\title{
Small-scale structure of the galactic cirrus emission ${ }^{\star}$
}

\author{
Cs. Kiss ${ }^{1,2}$, P. Ábrahám ${ }^{1,2}$, U. Klaas ${ }^{1}$, D. Lemke ${ }^{1}$, Ph. Héraudeau ${ }^{1}$, C. del Burgo ${ }^{1}$, and U. Herbstmeier ${ }^{1}$ \\ ${ }^{1}$ Max-Planck-Institut für Astronomie, Königstuhl 17, 69117 Heidelberg, Germany \\ 2 Konkoly Observatory of the Hungarian Academy of Sciences, PO Box 67, 1525 Budapest, Hungary
}

Received 18 March 2002 / Accepted 22 November 2002

\begin{abstract}
We examined the Fourier power spectrum characteristics of cirrus structures in 13 sky fields with faint to bright cirrus emission observed with ISOPHOT in the 90-200 $\mu$ m wavelength range in order to study variations of the spectral index $\alpha$. We found that $\alpha$ varies from field to field with $-5.3 \leq \alpha \leq-2.1$. It depends on the absolute surface brightness and on the hydrogen column density. We also found different spectral indices for the same sky region at different wavelengths. Longer wavelength measurements show steeper power spectra. This can be explained by the presence of dust at various temperatures, in particular of a cold extended component. For the faintest areas of the far-infrared sky we derive a wavelength-independent spectral index of $\alpha=-2.3 \pm 0.6$ for the cirrus power spectrum. The application of the correct spectral index is a precondition for the proper disentanglement of the cirrus foreground component of the Cosmic Far-Infrared Background and its fluctuations.
\end{abstract}

Key words. ISM: structure - infrared: ISM

\section{Introduction}

In the far-infrared the most significant component superimposed on the Extragalactic Background is the cirrus emission of the Galaxy originating from irregularly shaped interstellar clouds (Low et al. 1984). The correct determination of the Cosmic Far-InfraRed Background (CFIRB) requires a precise removal of this foreground component (Guiderdoni et al. 1997). The CFIRB is characterized by two parameters: the absolute level and the amplitude of its fluctuations. In recent years observations made by ISOPHOT (Lemke et al. 1996), the photometer on board the ISO satellite (Kessler et al. 1996) made the determination of the CFIRB fluctuations possible.

CFIRB fluctuations are expected to show the following properties (see e.g. Hauser \& Dwek 2001): (1) the spatial distribution corresponds to a flat power spectrum, (2) they are isotropic and (3) they have a positive constant value. In contrast, the fluctuations due to galactic cirrus emission scale up with absolute brightness and can be described by a multifractal structure, yielding a steep Fourier power spectrum. It is usually described by one main parameter, the spectral index $\alpha$ :

$P=P_{0} \times\left(f / f_{0}\right)^{\alpha}$

where $P$ is the Fourier power, $f$ is the absolute value of the two-dimensional spatial frequency and $P_{0}$ is the Fourier power at the spatial frequency $f_{0}$ (Gautier et al. 1992). Since cirrus

Send offprint requests to: Cs. Kiss, e-mail: pkisscs@konkoly.hu

^ Based on observations with ISO, an ESA project with instruments funded by ESA Member States (especially the PI countries: France, Germany, the Netherlands and the United Kingdom) and with the participation of ISAS and NASA. fluctuations are different from CFIRB fluctuations in at least two features (spatial frequency- and brightness-dependence), it is possible to separate them from each other in two independent ways:

(1) The decomposition through the brightness dependence at a fixed spatial frequency was performed by Kiss et al. (2001, hereafter Paper I), and resulted in a new determination of the CFIRB fluctuation amplitudes at 90 and $170 \mu \mathrm{m}$.

(2) The decomposition using the Fourier power spectrum was introduced by Guiderdoni et al. (1997). For faint fields, where CFIRB fluctuations are expected to be dominant in the highest spatial frequency range, the knowledge of the cirrus spectral index is mandatory for decomposition. Gautier et al. (1992) determined a spectral index of the galactic cirrus emission $\alpha \approx-3$ from the analysis of $100 \mu \mathrm{m}$ IRAS scans. For regions of faint total FIR brightness emission Lagache \& Puget (2000) and Matsuhara et al. (2000) derived CFIRB fluctuation amplitudes applying this spectral index of $\alpha \approx-3$ for the cirrus decomposition.

Herbstmeier et al. (1998) analysed a few fields observed by ISOPHOT in the $90-180 \mu \mathrm{m}$ wavelength range. They found a variation of spectral indices in the range of $-0.5 \geq \alpha \geq-3.6$, depending on wavelength, brightness and sky position. However, this sample was too small (four fields, two of them measured only at one wavelength) for general conclusions, but their results clearly indicated that the $\alpha=-3$ cirrus spectral index may not be universal.

Despite the strong impact of the cirrus power spectrum on the correct determination of the characteristics of the CFIRB, cirrus power spectrum properties were not yet analysed in 

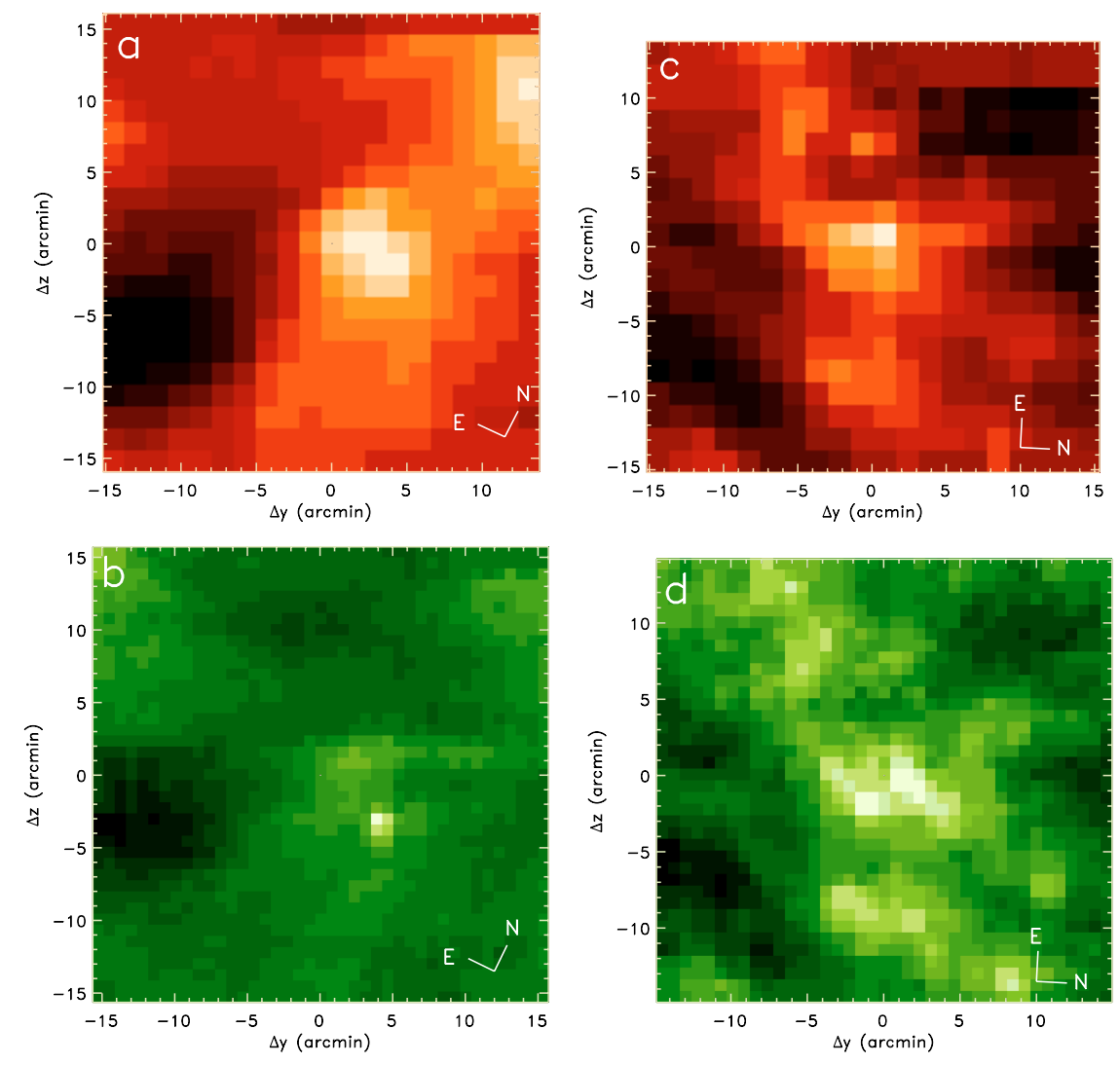

Fig. 1. 100 and $200 \mu \mathrm{m}$ range images of two sample fields in the satellite coordinate system (Laureijs et al. 2002). The central sky positions of the images are listed in Table 1 . The orientation of the celestial coordinate system is indicated. a) Cham-II, $200 \mu \mathrm{m} ; \mathbf{b}) \mathrm{Cham}-\mathrm{II}, 100 \mu \mathrm{m}$; c) NPC 1, $200 \mu \mathrm{m}$; d) NPC 1, $90 \mu \mathrm{m}$. While the FIR emission in NPC 1 arises from the same cirrus structure at both wavelengths, there seems to be an additional component of cold condensations in Cham II, as discussed in Sect. 5.1.

detail for $\lambda>100 \mu \mathrm{m}$ and at a resolution achievable by ISOPHOT. In this paper we analyse 13 sky regions measured by ISOPHOT in the $90-200 \mu \mathrm{m}$ range and investigate the characteristics of their Fourier power spectra.

\section{Observations and data analysis}

\subsection{Selection of ISOPHOT maps}

We selected 20 FIR maps from the ISO archive, covering 13 separate sky regions. All selected maps were obtained in the PHT22 staring raster observing mode (Laureijs et al. 2002). We excluded maps containing strong point sources or obvious spatial structures. This selection is a subsample of the fields used in Paper I. The selected maps had to show a clear sign of cirrus emission in the power spectrum. This requires a minimum average surface brightness of $\langle B\rangle \approx 3 \mathrm{MJysr}^{-1}$. Such fields are slightly brighter than the faintest fields in the far-infrared sky $\left(\langle B\rangle \approx 2 \mathrm{MJysr}^{-1}\right)$ in the $90-200 \mu \mathrm{m}$ wavelength range after the subtraction of the Zodiacal Light.

Our sample covers the surface brightness range 23-60 $\mathrm{MJysr}^{-1}$, ranging from faint to bright cirrus, and faint molecular cloud emission. The basic properties of the maps are summarized in Table 1. Images of two fields are shown in Fig. 1, more images are presented in Herbstmeier et al. (1998).

\subsection{Data reduction}

We used the final maps produced for the confusion noise analysis in Paper I. For a detailed description of the data reduction steps we refer to this paper. The data reduction comprised the following main steps:

- basic data analysis with PIA ${ }^{1}$ V8.2 (Gabriel et al. 1997) from ERD to AAP level;

- flat-fielding using first quartile normalization;

- subtraction of the Zodiacal emission.

\subsection{Fourier power spectrum}

Fourier transformation of the maps was performed by routines written in IDL $^{2}$ based on the standard IDL FFT routine with periodogram normalisation (Press et al. 1992). For all maps the highest spatial sampling frequency corresponds to the pixel size of the $\mathrm{C} 100$ and C200 array cameras. The Nyquist limit that allows correct sampling (without aliasing) is half of that frequency (Press et al. 1992, Chap. 12.1), i.e. the double pixel size of the array. Therefore, the upper limit

\footnotetext{
${ }^{1}$ PIA is a joint develpoment by the ESA Astrophysics Division and the ISOPHOT consortium led by the Max-Planck-Institut für Astronomie (MPIA), Heidelberg

${ }^{2}$ Interactive Data Language, Version 5.2 and 5.3, Research Systems Inc.
} 
Table 1. Basic observational and derived parameters of fields mapped by ISOPHOT. The columns are: (1) name of the field as specified by the observer; (2) central wavelength of filter; (3) map size; (4)-(5) center of the field in galactic coordinates; (6) median surface brightness from ISOPHOT photometry with Zodiacal Light subtracted; quoted standard deviations are a measure of the dynamic range of the map (7) spectral index of the power spectrum; (8) fluctuation power at the reference spatial frequency $1 / d_{0}\left(d_{0}=4^{\prime}\right)$; (9)-(10) parameters describing the relative strength of the instrument noise power spectrum to the signal power spectrum (see Eq. (2) for the definitions); (11) average neutral hydrogen column density (Dickey \& Lockman 1990); (12) ${ }^{12}$ CO integrated intensity (Dame et al. 2001); (13) associated objects or dark clouds located (at least partially) inside the field; (14) references with the description of the field.

\begin{tabular}{|c|c|c|c|c|c|c|c|c|c|c|c|c|c|}
\hline (1) & (2) & (3) & (4) & (5) & (6) & (7) & (8) & (9) & (10) & (11) & (12) & (13) & (14) \\
\hline \multirow[t]{2}{*}{ field } & $\lambda$ & size & \multicolumn{2}{|c|}{ field center } & $\left\langle\mathrm{B}_{\lambda}\right\rangle$ & $\alpha$ & $P_{0}$ & $\delta \alpha$ & $\delta P_{0}$ & $\mathrm{~W}\left({ }^{12} \mathrm{CO}\right)$ & $N(\mathrm{HI})$ & associated objects & references \\
\hline & $(\mu \mathrm{m})$ & (arcmin) & $l$ & $b$ & $\left(\mathrm{MJysr}^{-1}\right)$ & & $\left(\mathrm{Jy}^{2} \mathrm{sr}^{-1}\right)$ & & & $\left(\mathrm{K} \mathrm{kms}^{-1}\right)$ & $\left(\mathrm{cm}^{-2}\right)$ & & \\
\hline \multirow[t]{2}{*}{ NGP } & 180 & $46.0 \times 46.0$ & 88.9 & 73.0 & $3.1 \pm 0.1$ & $-2.53 \pm 0.22$ & $1.8 \times 10^{3}$ & 1.59 & 0.95 & - & $9.0 \times 10^{19}$ & North Galactic Pole & Juvela et al. (2000) \\
\hline & & & & & & & & & & & & & Herbstmeier et al. (1998) \\
\hline Draco & 90 & $7.7 \times 7.7$ & 89.8 & 38.6 & $5.7 \pm 0.3$ & $-2.45 \pm 0.17$ & $5.3 \times 10^{3}$ & 1.47 & 0.76 & - & $2.1 \times 10^{20}$ & Draco nebula & Herbstmeier et al. (1998) \\
\hline Draco & 170 & $27.6 \times 18.4$ & 89.8 & 38.6 & $4.7 \pm 0.5$ & $-4.09 \pm 0.22$ & $2.9 \times 10^{3}$ & 2.71 & 1.39 & - & $2.1 \times 10^{20}$ & Draco nebula & Herbstmeier et al. (1998) \\
\hline Cep & 90 & $14.7 \times 20.7$ & 108.0 & 13.6 & $23.5 \pm 3.1$ & $-3.42 \pm 0.15$ & $3.3 \times 10^{4}$ & 1.96 & 1.01 & 6.9 & $2.5 \times 10^{21}$ & Cepheus Flare & Herbstmeier et al. (1998) \\
\hline Cep & 170 & $21.5 \times 24.5$ & 108.0 & 13.6 & $68.2 \pm 12.7$ & $-4.19 \pm 0.12$ & $3.0 \times 10^{5}$ & 1.95 & 1.56 & 6.9 & $2.5 \times 10^{21}$ & Cepheus Flare & Herbstmeier et al. (1998) \\
\hline NPC1 & 90 & $29.9 \times 29.1$ & 121.6 & 24.2 & $7.6 \pm 0.8$ & $-3.10 \pm 0.04$ & $5.0 \times 10^{3}$ & 2.16 & 0.64 & 6.6 & $6.9 \times 10^{20}$ & Polaris Flare & Gautier et al. (1992) \\
\hline NPC1 & 200 & $30.7 \times 29.1$ & 121.6 & 24.2 & $26.1 \pm 5.3$ & $-3.46 \pm 0.09$ & $1.1 \times 10^{5}$ & 2.30 & 0.95 & 6.6 & $6.9 \times 10^{20}$ & Polaris Flare & Gautier et al. (1992) \\
\hline NPC2 & 90 & $29.9 \times 29.1$ & 122.0 & 24.6 & $7.4 \pm 0.7$ & $-2.83 \pm 0.09$ & $5.7 \times 10^{3}$ & 1.74 & 0.89 & 5.5 & $6.7 \times 10^{20}$ & Polaris Flare & Gautier et al. (1992) \\
\hline NPC2 & 200 & $30.7 \times 29.1$ & 122.0 & 24.6 & $22.6 \pm 3.8$ & $-3.39 \pm 0.08$ & $9.7 \times 10^{4}$ & 2.19 & 0.63 & 5.5 & $6.7 \times 10^{20}$ & Polaris Flare & Gautier et al. (1992) \\
\hline North1 & 90 & $29.9 \times 29.9$ & 100.0 & 14.8 & $13.3 \pm 1.4$ & $-3.05 \pm 0.09$ & $9.7 \times 10^{3}$ & 1.83 & 0.91 & 4.5 & $1.6 \times 10^{21}$ & LDN 1122 & Yonekura et al. (1997) \\
\hline North1 & 200 & $30.7 \times 30.7$ & 100.0 & 14.8 & $34.4 \pm 5.6$ & $-4.70 \pm 0.17$ & $4.5 \times 10^{4}$ & 2.43 & 0.52 & 4.5 & $1.6 \times 10^{21}$ & LDN 1122 & Yonekura et al. (1997) \\
\hline \multirow[t]{2}{*}{ North2 } & 90 & $29.9 \times 29.9$ & 108.0 & 15.2 & $12.4 \pm 1.8$ & $-3.65 \pm 0.13$ & $9.2 \times 10^{3}$ & 1.42 & 0.90 & 6.6 & $2.1 \times 10^{21}$ & LDN 1147,1148 & Lee et al. (2001) \\
\hline & & & & & & & & & & & & & Yonekura et al. (1997) \\
\hline \multirow[t]{2}{*}{ North2 } & 200 & $30.7 \times 30.7$ & 108.0 & 15.2 & $40.7 \pm 12.5$ & $-3.81 \pm 0.09$ & $3.2 \times 10^{5}$ & 1.94 & 0.61 & 6.6 & $2.1 \times 10^{21}$ & LDN 1147,1148 & Lee et al. (2001) \\
\hline & & & & & & & & & & & & & Yonekura et al. (1997) \\
\hline \multirow[t]{2}{*}{ M01 } & 180 & $27.6 \times 27.6$ & 100.0 & 30.6 & $5.0 \pm 0.2$ & $-2.56 \pm 0.41$ & $2.3 \times 10^{2}$ & 0.89 & 0.75 & - & $4.2 \times 10^{20}$ & faint field in Dra & Herbstmeier et al. (1998) \\
\hline & & & & & & & & & & & & & Herbstmeier et al. (1997) \\
\hline M03 & 180 & $27.6 \times 27.6$ & 117.6 & 46.1 & $3.7 \pm 0.1$ & $-2.12 \pm 0.23$ & $5.8 \times 10^{2}$ & 0.82 & 0.71 & - & $1.5 \times 10^{20}$ & faint field in UMi & Ábrahám et al. (1997) \\
\hline \multirow[t]{2}{*}{ TMC2-1 } & 200 & $30.7 \times 30.7$ & 173.9 & -15.7 & $56.5 \pm 8.9$ & $-5.26 \pm 0.15$ & $3.7 \times 10^{5}$ & 1.74 & 0.86 & 18.9 & $1.7 \times 10^{21}$ & LDN 1529, 1531 & Lee et al. (2001) \\
\hline & & & & & & & & & & & & Taurus 2 mol. cl. & Chappell \& Scalo (2001) \\
\hline \multirow[t]{2}{*}{ TMC2-2 } & 200 & $30.7 \times 30.7$ & 174.3 & -15.9 & $63.7 \pm 8.5$ & $-3.49 \pm 0.14$ & $3.8 \times 10^{5}$ & 1.66 & 1.04 & 16.3 & $1.8 \times 10^{21}$ & LDN 1529, 1531 & Lee et al. (2001) \\
\hline & & & & & & & & & & & & Taurus 2 mol. cl. & Chappell \& Scalo (2001) \\
\hline Cha1S & 100 & $19.9 \times 19.9$ & 297.3 & -16.2 & $29.3 \pm 3.2$ & $-3.80 \pm 0.08$ & $1.4 \times 10^{5}$ & 2.47 & 0.79 & 10.3 & $9.2 \times 10^{20}$ & Chamaeleon mol. cl. & Tóth et al. (2000) \\
\hline ChamII & 100 & $31.4 \times 31.4$ & 303.5 & -14.2 & $23.9 \pm 3.3$ & $-2.95 \pm 0.08$ & $1.7 \times 10^{5}$ & 0.91 & 0.89 & 16.1 & $1.2 \times 10^{21}$ & DCld 303.3-14.3, & Tóth et al. (2000) \\
\hline ChamII & 200 & $30.7 \times 30.7$ & 303.5 & -14.2 & $38.4 \pm 14.6$ & $-5.17 \pm 0.18$ & $2.4 \times 10^{5}$ & 2.48 & 0.53 & 16.1 & $1.2 \times 10^{21}$ & 303.5-14.4 & Tóth et al. (2000) \\
\hline
\end{tabular}

of the spatial frequency considered for the Fourier power spectrum was set to the Nyquist-limit, which is $\theta_{\min }=92^{\prime \prime}$ for images with $\lambda \leq 100 \mu \mathrm{m}$ (C100 detector) and $\theta_{\min }=184^{\prime \prime}$ for images with $\lambda>100 \mu \mathrm{m}$ (C200 detector). Since the pixel sizes of the ISOPHOT C100 and C200 cameras were adjusted to the diffraction limit, this limit should be kept, even if the raster sampling was finer than the pixel size.

Instead of averaging $P(f)$ fluctuation power values in annuli for each $f=|\boldsymbol{f}|$ spatial frequency, we used all individual data points ( $f, P(f)$ pairs) to derive the final power spectrum. The spectral index $\alpha$ is derived by robust line fitting to all data points in the $\log (f)-\log (P)$ space.

In most cases the whole power spectrum could be satisfactorily fitted by one spectral index. For some faint fields, however, the high frequency end of the spectrum showed a different (flatter) spectral index, due to a higher structure noise level. In such cases we only used the low and mid frequency part to derive $\alpha$. The reason for this flattening will be discussed below in Sect. 4.

\section{Features affecting the power spectrum}

There are several contributions to the power spectrum that can affect its final shape. We analyse the effects of the instrument noise, a point-source footprint and an extended source profile on the final power spectrum. The correct separation of the power spectrum components requires well-distinguishable spectral indices, otherwise they cannot be disentangled.

\subsection{Power spectrum of the instrument noise}

Instrument noise can be a significant contribution to the fluctuation power at low surface brightness and high spatial frequencies. As discussed in detail in Paper I, the most representative estimates of the instrument noise are PIA-noise (which reflects the statistical uncertainties in signal derivation) and flat-field noise (which reflects the variation of the signal from individual detector pixels at the same sky position). Flat-field noise can only be determined for oversampled maps. 

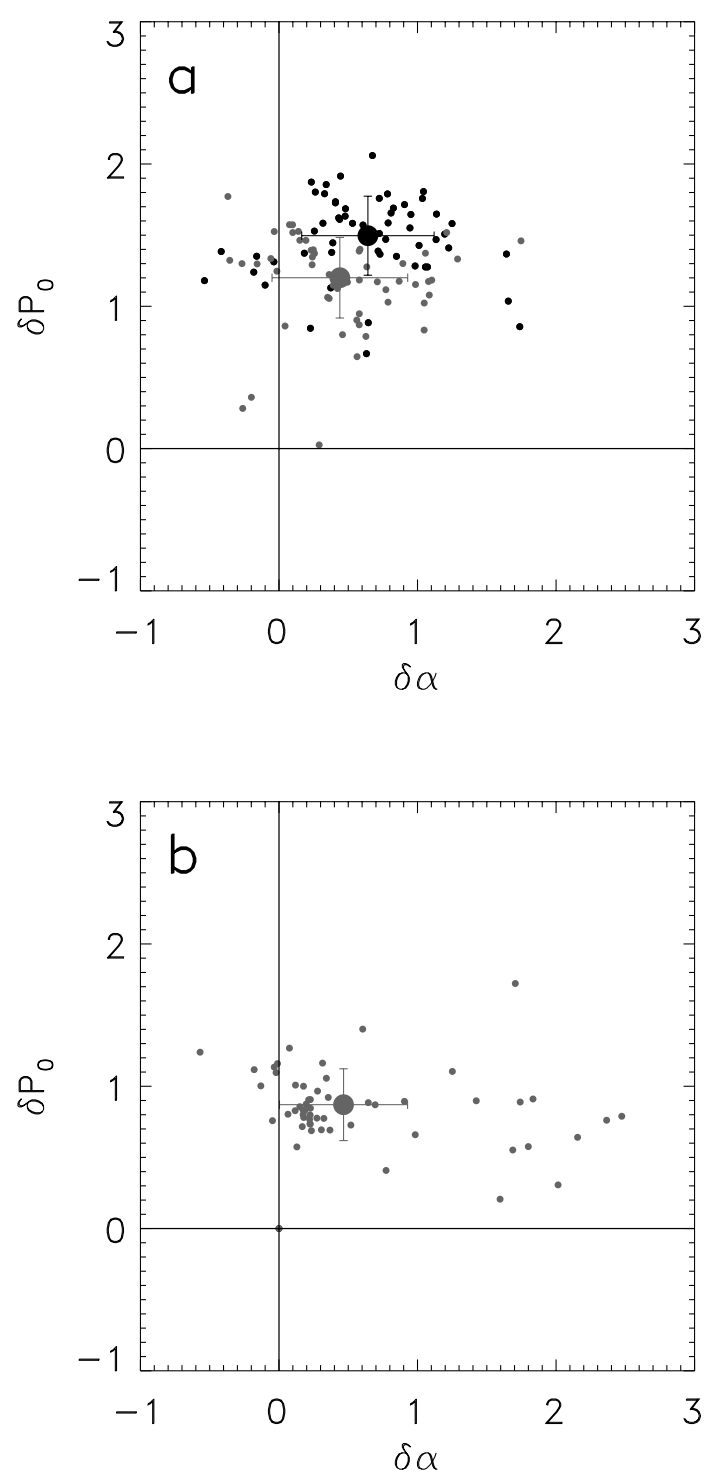

Fig. 2. Relative strength of the instrument noise - to the signal power spectrum. a) Subsample of 56 maps from Paper I, observed with the C200 detector in oversampling mode. Black dots: flat-field noise; gray dots: PIA-noise. b) Subsample of 60 maps from Paper I, observed with the $\mathrm{C} 100$ detector. In this case no representative values of the flat-field noise could be dervied due to the lack of a sufficient number of oversampled maps. The average values and the standard deviations both in $\delta \alpha$ and $\delta P_{0}$ are marked via large dots and error bars, respectively.

We assume that both the signal and instrument noise power spectra - derived from the surface brightness maps and the corresponding noise maps, respectively - are described by a power law behaviour as in Eq. (1). Here the $f_{0}$ values correspond to the Nyquist-limits, i.e. $\theta_{\min }=92^{\prime \prime}$ and $184^{\prime \prime}$ for the C100 and C200 detectors, respectively.

The signal power spectrum is not affected by the instrument noise, if the power spectrum of the instrument noise is slightly weaker than that of the signal in the frequency range analysed. This is ensured if two criteria are fulfilled simultaneously: (1) the fluctuation power of the instrument noise $P_{\mathrm{i}}$ is lower by at least a factor of 2 than that of the signal $P$ s at the resolution limit, $\theta_{\min }$ and (2) the power spectrum of the signal is steeper than that of the instrument noise. These conditions are parametrized by the following two quantities ( $\mathrm{i}$ and $\mathrm{s}$ denote instrument noise and signal, respectively):

$\delta \alpha=\alpha_{\mathrm{i}}-\alpha_{\mathrm{s}}>0 \quad \delta P_{0}=\log _{10}\left(\frac{P_{0}^{\mathrm{s}}}{P_{0}^{\mathrm{i}}}\right)>0.3$.

For the instrument noise analysis we used a larger sample of maps than for the cirrus power spectrum study. In Table 1 we provide the respective values of $\delta \alpha$ and $\delta P_{0}$ for our special fields, which can be compared with the ranges of $\delta \alpha$ and $\delta P_{0}$ for the larger sample of maps.

In the case of the $\mathrm{C} 200$ detector we calculated $\delta \alpha$ and $\delta P_{0}$ for a subsample of 56 maps from Paper I, observed with full oversampling (Fig. 2a).

Most of the flat-field and PIA-noise data points are located in the quadrant where both $\delta \alpha$ and $\delta P_{0}$ are positive. Although there are a few points in the negative $\delta \alpha$ regime, they are close to zero, representing nearly parallel instrument noise and signal power spectra. Since in all cases $\delta P_{0}>0.3$, the instrument noise power spectrum does not exceed the signal power spectrum for frequencies below $\theta_{\min }^{-1}$. The data points with $\delta \alpha \lesssim 0$ belong to the faintest regions of the sky with a nearly flat power spectrum, showing no signs of cirrus $\left(\alpha_{\mathrm{s}}\right.$ and $\left.\alpha_{\mathrm{i}} \approx 0\right)$. The distributions of the PIA- and flat-field noise data points are similar, and the average values are within the standard deviations in $\delta \alpha$ and $\delta P_{0}$ (big black and gray dots with error bars in Fig. 2a). This demonstrates that the method of noise determination has no impact on the final result.

In the case of the $\mathrm{C} 100$ detector it was not possible to perform a similar investigation due to the lack of suitable oversampled maps. Therefore, we analysed 60 observations from Paper I by calculating the PIA-noise values only (Fig. 2b). Also the $\mathrm{C} 100$ detector measurements fulfil the requirements of a suitable signal-to-noise ratio.

This noise analysis justifies that for both C200 and C100 maps of Table 1 instrument noise does not affect the final shape of the power spectrum, in particular since for all of them $\delta \alpha>0.8$ and $\delta P_{0}>0.5$.

\subsection{Power spectra of point- and extended sources}

In Fig. 3a we present a model cirrus spectrum with an expected cirrus spectral index of $\alpha=-3$ convolved with the $90 \mu \mathrm{m}$ and $200 \mu \mathrm{m}$ theoretical footprints (footprint = convolution of the telescope point spread function with the pixel aperture, Laureijs et al. 2002). This figure shows that the footprint has only a slight effect below the actual Nyquist limit, and this effect can be neglected when deriving the spectral index. A strong point source may dominate the whole spectrum, but maps containing these features were not included in our sample.

As will be shown in Sect. 5.1, extended sources with excess emission may be superimposed on the cirrus-like intensity distribution. We tested the effect of these sources on the power spectrum assuming a Gaussian shape, and a $F W H M$ of $1 / 3$ and $1 / 6$ of the size of the original map, respectively, as presented in Fig. 3b. The shape of the resulting power spectrum 

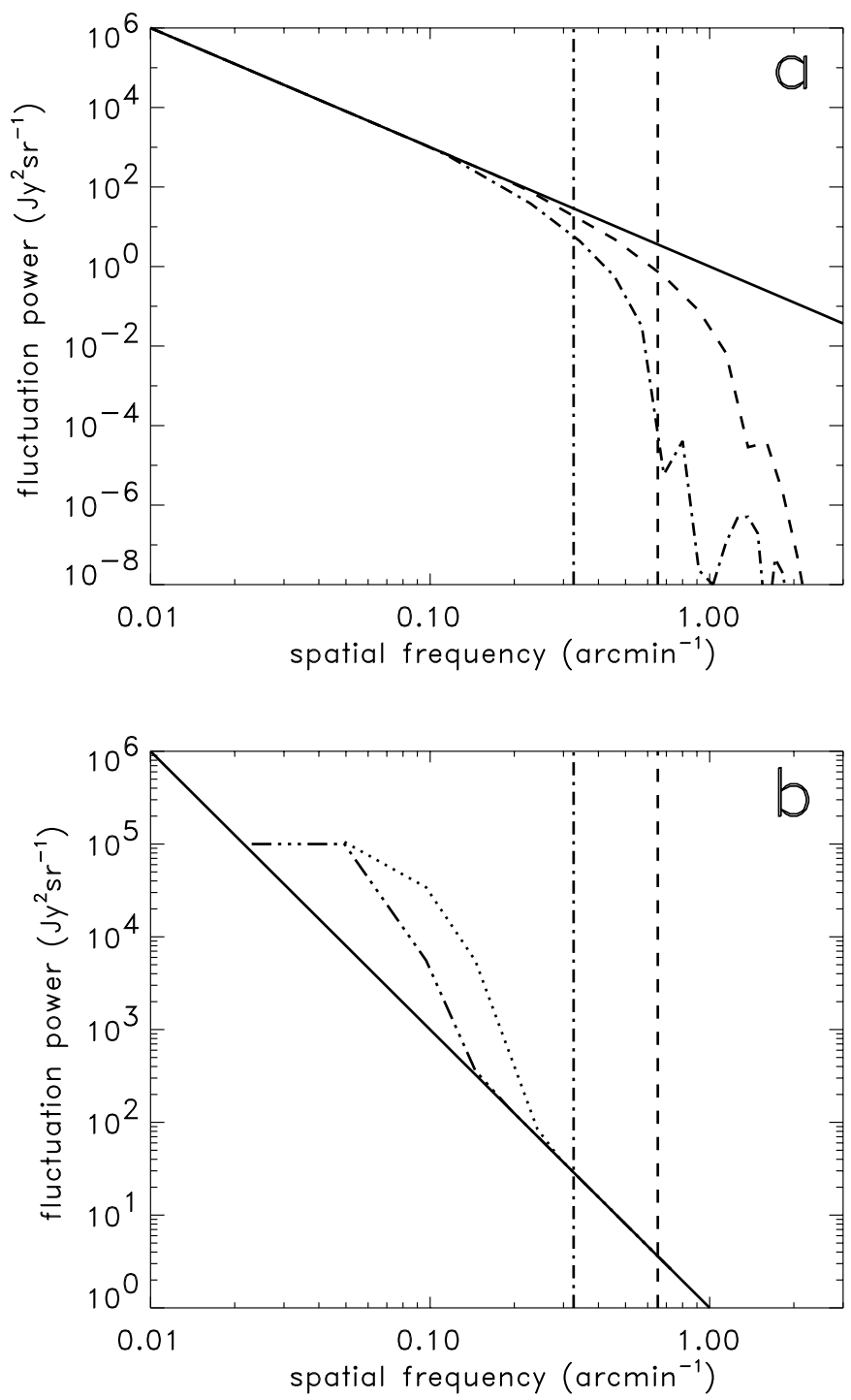

Fig. 3. Schematic representation of the contribution of different emission components to the final power spectrum. a) Convolution of a theoretical cirrus spectrum of arbitrary stength $\left(\alpha=-3, P=10^{3} \mathrm{Jy}^{2} \mathrm{sr}^{-1}\right.$ at $f=0.1 \operatorname{arcmin}^{-1}$ ) with ISOPHOT's theoretical point-source footprint at $90 \mu \mathrm{m}$ (dashed line) and at $200 \mu \mathrm{m}$ (dash-dotted line). The pure, non-convolved cirrus spectrum is also shown (solid line). b) Resulting power spectrum of a cirrus-like intensity distribution $(\alpha=-3$, solid line) and an extended source of Gaussian shape with a $F W H M$ of $1 / 6$ (dotted line) and 1/3 (dash-triple-dotted line) of the size of the original map. The Gaussians are normalized to $P=10^{5} \mathrm{Jy}^{2} \mathrm{sr}^{-1}$ at $f=0 \operatorname{arcmin}^{-1}$. The Nyquist-limits for the C100 and C200 filter maps are marked both in a) and b) by vertical dashed and dash-dotted lines, respectively.

strongly depends on the relative strength of the cirrus and extended source components. The typical effect of the extended source is to cause a "hump" at medium spatial frequencies, if the strengths of the two components are comparable. Similar (but weaker) features can be identified in some of our real power spectra, especially at $200 \mu \mathrm{m}$ (see Fig. 4). The high frequency part of an extended source power spectrum is rather steep and therefore - if dominant - can lead to a power spectrum steeper than that of pure cirrus. A set of extended sources has a power spectrum similar to that of a single one, but the higher the number of sources, the shallower the final power spectrum. In certain configurations these spectra may be described by an average spectral index of $\alpha \approx-3$, leading to a spectrum, which is in practice undistinguishable from the one of cirrus.

\section{Results}

Table 1 and Fig. 4 present the results of the power spectrum analysis. The fields in our analysis were characterized by the following physical parameters: median FIR surface brightness from ISOPHOT photometry with Zodiacal Light subtracted (this work), neutral hydrogen column density (Dickey \& Lockman 1990) and ${ }^{12} \mathrm{CO}$ integrated intensity (Dame et al. 2001). The power spectra follow quite well the expected powerlaw behaviour up to the spatial frequency corresponding to the resolution limit, and therefore can be satisfactorily fitted by one single spectral index $\alpha$. The only exceptions are the fields NGP (Fig. 4f), M01 (Fig. 4e) and M03 at $180 \mu \mathrm{m}$. They show a flattening of the spectrum for the highest spatial frequencies which was eventually excluded in the derivation of the $\alpha$ values given in Table 1.

We suggest that the relatively strong structure noise at high spatial frequencies arises from the fluctuations due to the extragalactic background. As discussed in Paper I, the extragalactic background is the major contributor to the structure noise at high spatial frequencies for the faintest regions of the farinfrared sky measured with the $\mathrm{C} 200$ detector. These three faint fields have an average surface brightness comparable to that of the cosmological fields. The extragalactic background is expected to have a fluctuation power of $\sim 8 \times 10^{2} \mathrm{Jy}^{2} \mathrm{sr}^{-1}$ at $170 \mu \mathrm{m}$ with our FFT normalization. Fluctuation powers at the reference spatial frequency listed in Table 1 (Col. 8) are fitted values using the cirrus-like power spectrum, therefore they can be lower than the expected CFIRB fluctuation level.

We calculated the median values of the spectral indices for the $\mathrm{C} 100$ and $\mathrm{C} 200$ fields $\left(\bar{\alpha}_{\mathrm{C} 1}\right.$ and $\bar{\alpha}_{\mathrm{C} 2}$, respectively), in order to compare them with previous results. This resulted in $\bar{\alpha}_{\mathrm{C} 1}=-3.15 \pm 0.48$ and $\bar{\alpha}_{\mathrm{C} 2}=-3.87 \pm 1.06$. The median $\mathrm{C} 100$ spectral index is not much different from the one determined by Gautier et al. (1992) from the analysis of IRAS $100 \mu \mathrm{m}$ scans, however it shows a relatively large scatter. Abergel et al. (1996) performed a pixelwise determination of the spectral index for a $12.5 \times 12.5$ ISSA map close to the South Ecliptic Pole. At $100 \mu \mathrm{m}$ they obtained an average spectral index of $\bar{\alpha}_{100}=$ -3.34 (using the relation $\alpha=-2(\beta-1)$ between their $\beta$-value and our spectral index). The distribution of the spectral index over the field had a Gaussian shape with $\sigma_{\alpha}=0.36$. This means, that within one field the variation is comparable to the variation we found for our sample fields. The C200 spectral indices are significantly steeper with an even higher scatter than the C100 spectral indices.

Spectral indices show a clear dependence both on the surface brightness and on the neutral hydrogen column density. Brighter fields and fields with higher HI column density usually have a steeper power spectrum (Fig. 5). We tested the degree of correlation by calculating the linear Pearson 


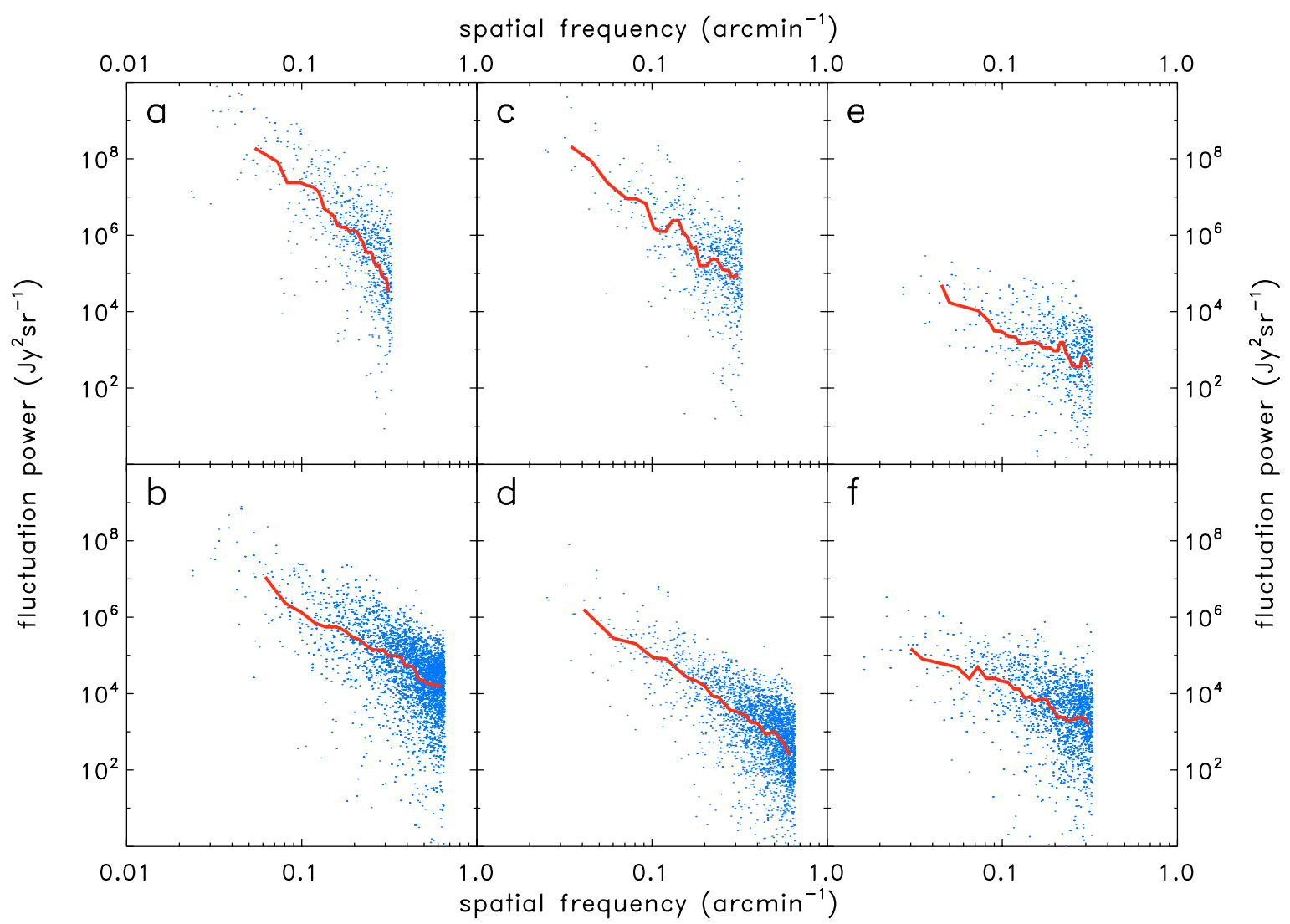

Fig. 4. Power spectra of six sample fields, two of them observed by both the $\mathrm{C} 100$ and the $\mathrm{C} 200$ camera. The images corresponding to the first four power spectra are presented in Fig. 1. Images of M01 and NGP can be found in Herbstmeier et al. 1998. a) Cham-II, $200 \mu \mathrm{m}$; b) Cham-II, $100 \mu \mathrm{m}$; c) NPC 1, $200 \mu \mathrm{m}$; d) NPC 1, $90 \mu \mathrm{m}$; e) M01, $180 \mu \mathrm{m}$; f) NGP, $180 \mu \mathrm{m}$. Each dot represents a $(f, P(f))$ pair, the solid line is smoothed average to outline the trend. The high frequency end is determined by the Nyquist-limit, the low frequency end by the size of the map.

correlation coefficient which resulted in $0.69,0.80$ and 0.67 for the $\log _{10}\left\langle B_{\mathrm{C} 1}\right\rangle-\alpha_{\mathrm{C} 1}, \log _{10}\left\langle B_{\mathrm{C} 2}\right\rangle-\alpha_{\mathrm{C} 2}$ and $\log _{10} \mathrm{~N}(\mathrm{HI})-\alpha$ relations, respectively, without the data points of the Draco region (marked in Fig. 5 by an arrow).

If we exclude in Fig. $5 \mathrm{~b}$ the three data points with high $B_{\mathrm{C} 2}$ and $\alpha<-4.5$, then the $\log _{10}\left\langle B_{\mathrm{C} 2}\right\rangle-\alpha_{\mathrm{C} 2}$ correlation is much tighter with a linear Pearson correlation coefficient of 0.94 . This exclusion is justified by the fact, that all three fields have a high HI column density and two of them show a large $\alpha_{\mathrm{C} 2} / \alpha_{\mathrm{C} 1}$ ratio (Fig. 5c), indicative of a relatively large molecular hydrogen content, as discussed below in Sect. 5.2.

It is possible to determine an $\alpha_{\mathrm{C} 2}$ value for the faintest fields of the far-infrared sky, by a robust linear extrapolation of the $\log _{10}\left\langle B_{\mathrm{C} 2}\right\rangle-\alpha_{\mathrm{C} 2}$ relation. In order to account for the contribution by the CFIRB the $B_{\mathrm{C} 2}$ values were reduced beforehand by $0.8 \mathrm{MJysr}^{-1}$ (Paper I). For all points except the Draco region this resulted in $\alpha_{\mathrm{C} 2}=-2.3 \pm 0.6$ for the faintest cirrus fields $\left(\langle B\rangle \approx 2 \mathrm{MJysr}^{-1}\right)$. Excluding in addition the three points with $\alpha<-4.5, \alpha_{\mathrm{C} 2}=-2.1 \pm 0.4$. The impact of this power law in separating the CFIRB from the galactic cirrus is discussed in Sect. 5.3.

In Fig. 6 we plot the ratio of the spectral indices $\alpha_{\mathrm{C} 2} / \alpha_{\mathrm{C} 1}$ determined both for a C200 and a C100 map of the same field. Assuming that the FIR radiation at 100 and $200 \mu \mathrm{m}$ is emitted by the same structures the ratio $\alpha_{\mathrm{C} 2} / \alpha_{\mathrm{C} 1}$ should be $\approx 1$. It is obvious, that $\alpha_{\mathrm{C} 2} / \alpha_{\mathrm{C} 1}$ varies in the range 1.0-1.8. Since the determination of the spectral index is quite accurate (see Table 1), this deviation cannot be explained by measurement uncertainties. We propose an explanation in Sect. 5.1

\section{Discussion}

\subsection{Wavelength dependence of the spectral index}

Galactic cirrus emission is expected to have a spectral energy distribution of a modified black body, with $v^{2}$ emissivity law, and a dust colour temperature of $17.5 \pm 1.5 \mathrm{~K}$ (Lagache et al. 1998). If the FIR emission of a certain cloud can be well described by a single dust temperature, then the emission measured at two separate wavelengths should be strongly correlated, showing the same spatial structure and the same power spectrum. On the other hand, the presence of another component with different colour temperature can lead to an excess intensity at one wavelength. This yields also a different spatial structure of the emission, and therefore different power spectra at two wavelengths. Indications for such a dichotomy in cloud hierarchy were found by Abergel et al. (1996) from their analysis of the 60 and $100 \mu \mathrm{m}$ IRAS maps of the South Ecliptic Pole field. Uncorrelated features come from the coldest regions of the analysed fields. 

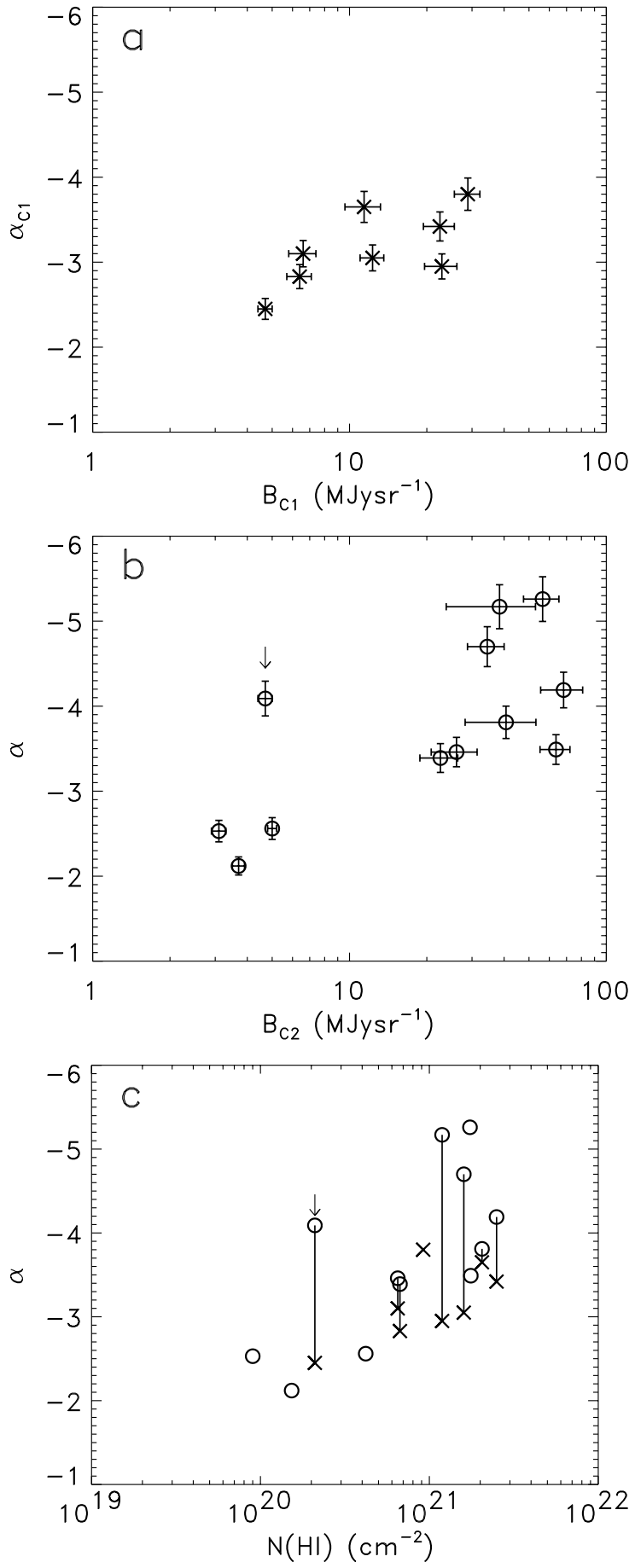

Fig. 5. Relationship of the spectral index with a) the $100 \mu \mathrm{m}$ range $(90-100 \mu \mathrm{m})$ median surface brightness $B_{\mathrm{Cl}}$, b) the $200 \mu \mathrm{m}$ range (170-200 $\mu \mathrm{m}$ ) median surface brightness $B_{\mathrm{C} 2}$, c) the average neutral hydrogen column density. Crosses and open circles mark fields observed at $\lambda \leq 100 \mu \mathrm{m}$ and $170 \leq \lambda \leq 200 \mu \mathrm{m}$, respectively. The data points of the Draco $170 \mu \mathrm{m}$ field are marked by arrows. Error bars in a) and b) represent the standard deviations in $B_{\mathrm{C} 1}$ and $B_{\mathrm{C} 2}$ and they mark the errors in fitting the spectral index in $\alpha$.

We tested the uniqueness of the colour temperatures for fields, where measurements at two wavelengths were available. The colour temperature is represented by the flux ratio of the two wavelengths. Two examples are presented in Fig. 7.

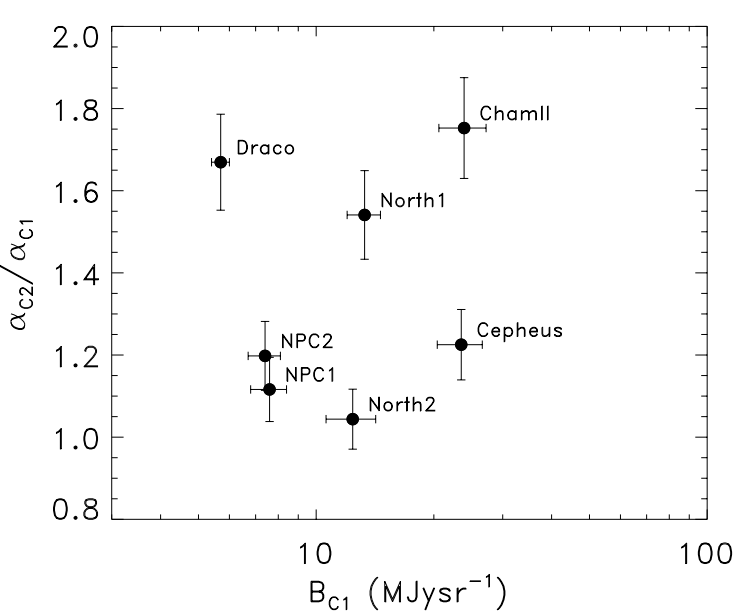

Fig. 6. Ratio of the spectral indices of sky regions measured in both a long wavelength (170-200 $\left.\mu \mathrm{m}, \alpha_{\mathrm{C} 2}\right)$ and at short wavelength (90-100 $\left.\mu \mathrm{m}, \alpha_{\mathrm{C} 1}\right)$ filter versus the average surface brightness in the $\mathrm{C} 100$ filter $\left(90\right.$ or $\left.100 \mu \mathrm{m}, B_{\mathrm{C} 1}\right)$. The regions are labelled by the names as given by the observer and as listed in Table 1 .

The 90-100 $\mu \mathrm{m}$ surface brightness maps were smoothed to the resolution of the 170-200 $\mu \mathrm{m}$ measurements in order to produce $B_{\mathrm{C} 1}-B_{\mathrm{C} 2}$ pixel-to-pixel scatter plots, where $B_{\mathrm{C} 1}$ and $B_{\mathrm{C} 2}$ are the surface brightnesses of the $90-100 \mu \mathrm{m}$ and $170-200 \mu \mathrm{m}$ map pixels, respectively. In most cases the scatter plot showed a well correlated $B_{\mathrm{C} 1}-B_{\mathrm{C} 2}$ emission and could be fitted by one straight line, corresponding to one single dust temperature. Using this relation we derived an excess surface brightness $\Delta B$ map, by subtracting the scaled up $B_{\mathrm{C} 1}$ emission from $B_{\mathrm{C} 2}$.

$\Delta B=B_{\mathrm{C} 2}-\left[A_{1} \times B_{\mathrm{C} 1}+A_{0}\right]$

where $A_{1}$ and $A_{0}$ are the slope and the interception of the linear fit to the scatter plot, respectively.

However, as presented in Fig. 7a, in some cases there was more than one characteristic colour temperature present in a field. The most conspicuous example is the Cham II field (gray dots in Fig. 7a). In this case, after removing the cirrus contribution according to the procedure of Eq. (3), the excess surface brightness image $\Delta B$ still showed the steep index of the original map. The steep $\Delta B$ power spectrum can be associated to a bright extended source, as suggested by the examples in Sect 3.2 and, indeed, the region contains dark cloud cores, namely DCld 303.3-14 and DCld 303.5-14.4 (see Table 1). The expected flattening at low spatial frequencies due to the extended source power spectrum (see Sect. 3.2) can also be identified. The characteristic size of the extended source derived from the power spectrum agrees well with the typical cold core extent of $\sim 10^{\prime}$ found by Tóth et al. (2000) in Chamaeleon, with temperatures of $\leq 15 \mathrm{~K}$. A similar effect can be observed for the North 1 field, however, the differences are smaller than in the case of the Cham II region. The feature in the power spectrum can be identified with the molecular $\left({ }^{13} \mathrm{CO}\right)$ core of the dark cloud LDN 1122 (Yonekura et al. 1997). In the case of the NPC 1 field we found that the power spectrum of the excess surface brightness image is significantly shallower than that of the $200 \mu \mathrm{m}$ surface brightness image, because this lacks any dark cloud core. 

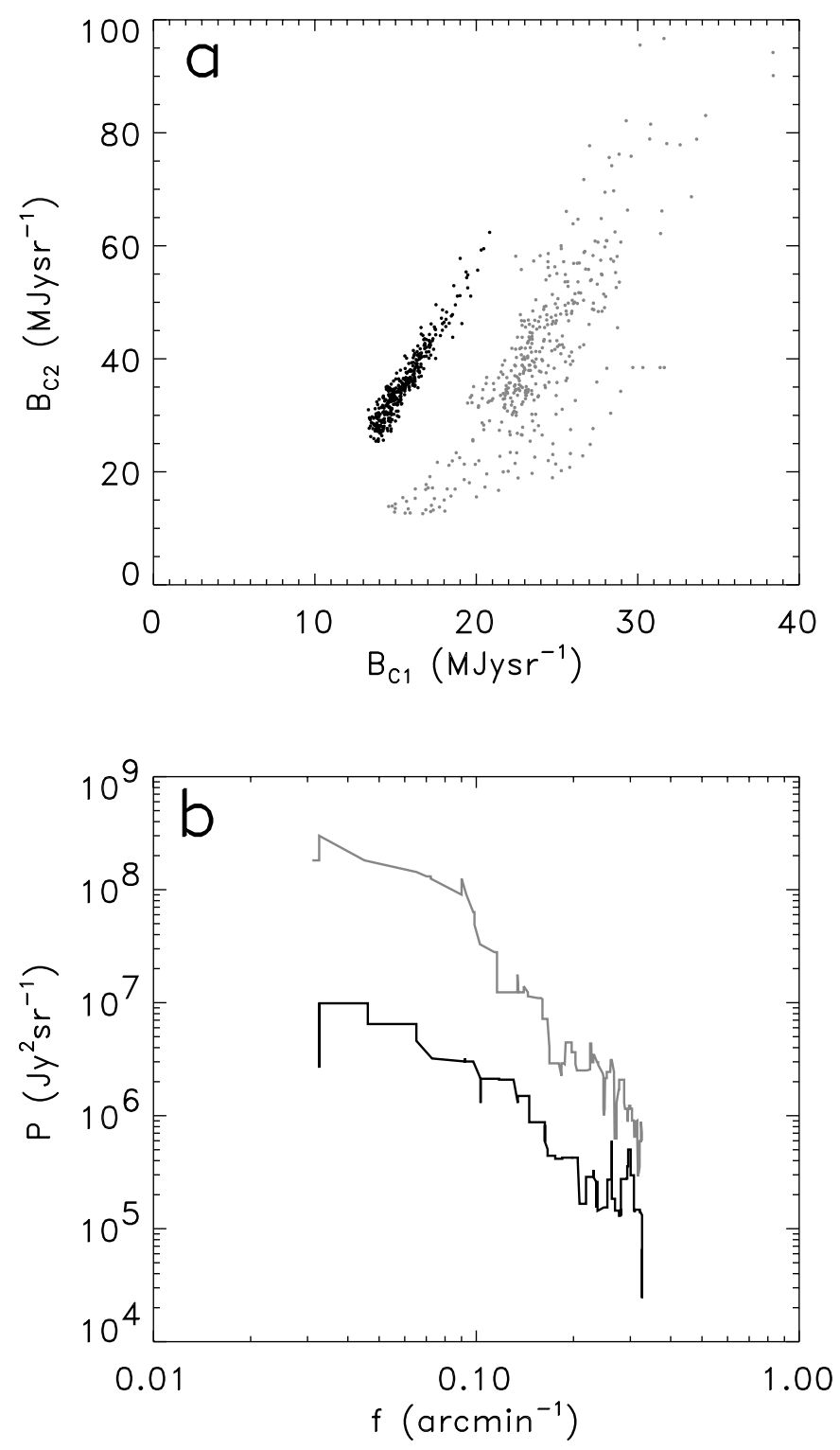

Fig. 7. Examples to demonstrate the effect of a non-uniform color temperature within the same field. a) C100 vs. C200 surface brightness scatter plots. The slope is closely related to the color temperature (see text for details). Black and gray dots represent the data points of the North 1 and Cham II fields, respectively. b) Power spectra of the $\Delta B$ images (cirrus component subtracted) of Cham II (gray) and North 1 (black).

No colour temperature could be derived for the Draco region via the scatter plot procedure, due to its small spatial extent and narrow surface brightness range. However, the presence of a relatively bright extended source is obvious in the $170 \mu \mathrm{m}$ image (see Herbstmeier et al. 1998). This produces very likely the very steep power spectrum observed at this wavelength, despite the low average surface brightness (see Fig. 5), as already mentioned by Herbstmeier et al. (1998).

These findings suggest that the wavelength dependence of the spectral indices is caused by the combined effects of non-uniform dust temperatures and the presence of extended sources in the fields we selected for our study. The separation of the cirrus and extended source components in the power spectra can be more easily performed by including spatial scales larger than the characteristic extent of our maps. At $170 \mu \mathrm{m}$ such an analysis would be possible by utilizing the ISOPHOT Serendipity Survey slews with a typical length of a few degrees (Bogun et al. 1996).

\subsection{The effect of atomic-to-molecular phase transition}

The correlation of the spectral indices with surface brightness and with neutral hydrogen column density is obvious for faint fields, while a scatter in $\alpha$ is observed for bright and high HI column density fields, as presented in Fig. 5. These fields also show strong molecular $\left({ }^{12} \mathrm{CO}\right)$ emission, as presented in Table 1. This large scatter can be observed for $N(\mathrm{HI}) \geq 10^{21} \mathrm{~cm}^{-2}$, which is approximately the column density of the atomic-to-molecular phase transition in the galactic cirrus (de Vries \& van Dishoek 1988; Meyerdierks et al. 1990). In these clouds the ratio of molecular hydrogen column density to atomic hydrogen column density may vary in the range of $\mathrm{N}\left(\mathrm{H}_{2}\right) / \mathrm{N}(\mathrm{HI}) \approx 0.2-5.0$ (Magnani et al. 1985). Therefore clouds showing the same $\mathrm{N}(\mathrm{HI})$ may have different total hydrogen masses. This diverse molecular content - and therefore diverse final structure - can lead to the observed scatter in the spectral indices.

\subsection{Implication of cirrus power spectra on the CFIRB fluctuations}

The spectral index for faint fields observed in the $170-200 \mu \mathrm{m}$ wavelength range is greater than the usually assumed $\alpha=-3$, which was originally derived by Gautier et al. (1992) from $100 \mu \mathrm{m}$ IRAS scans of medium-brightness cirrus fields. Due to the lack of a better determination this was widely applied to the faintest fields of the far-infrared sky and for longer wavelengths (e.g. Guiderdoni et al. 1997; Lagache \& Puget 2000). We showed that brighter extended sources - which are often present in cirrus fields - may significantly increase the steepness of the power spectrum. Therefore it is plausible, that the $\alpha=-3$ power law found for medium surface brightness fields results from the superposition of the power spectrum of a less steeper fractal structure with extended sources. Our analysis provides $\alpha=-2.3 \pm 0.6$ for the spectral index of the faintest cirrus fields. Although there is a discrepancy between $\alpha$-values determined at different wavelengths for brighter fields, the spectral indices should be the same for faint fields, since these lack additional extended sources and multiple dust colour temperatures. We finally conclude that a uniform $\alpha=-2.3 \pm 0.6$ cirrus spectral index - independent of wavelength - has to be applied for the faintest areas $\left(\langle B\rangle \approx 2 \mathrm{MJysr}^{-1}\right)$ of the farinfrared sky. The application of this $\alpha$-value would decrease the CFIRB fluctuation amplitudes obtained by previous works in the $170-200 \mu \mathrm{m}$ range by $5-20 \%$ (Kiss et al. 2002, in preparation). For somewhat brighter regions it is mandatory to determine the proper spectral index from the properties of the local structures. 


\section{Conclusions}

We examined the Fourier power spectrum characteristics of 13 sky fields with faint to bright cirrus emission in the 90$200 \mu \mathrm{m}$ wavelength range and derived the spectral index of the power spectrum, $\alpha$. We found that $\alpha$ varies from field to field. It has a clear dependence on the absolute surface brightness and on the hydrogen column density of the field. For longer wavelengths $(\lambda>100 \mu \mathrm{m})$ the scatter in $\alpha$ is larger, especially for higher hydrogen column densities. This is because for $N(\mathrm{HI}) \geq 10^{21} \mathrm{~cm}^{-2}$ the atomic-to-molecular phase transition leads to various $\alpha$ values especially in the 170-200 $\mu \mathrm{m}$ range, where the diverse molecular content is easier to observe. We also found a significant difference in spectral indices for the same sky region at different wavelengths. At longer wavelengths $(\lambda>100 \mu \mathrm{m})$ the power spectra are steeper. This can be explained by the coexistence of dust components with various temperatures within the same field and cold extended emission features which significantly affect the power spectrum. A spectral index of the cirrus power spectrum with a value of $\alpha=$ $-2.3 \pm 0.6$ - independent of wavelength - could be derived for the faintest areas of the far-infrared sky. The precise determination of the spectral index for each field will enable to correctly disentangle the galactic foreground components of the CFIRB.

Acknowledgements. The development and operation of ISOPHOT were supported by MPIA and funds from Deutsches Zentrum für Luft- und Raumfahrt (DLR). The ISOPHOT Data Center at MPIA is supported by Deutsches Zentrum für Luft- und Raumfahrt e.V. (DLR) with funds of Bundesministerium für Bildung und Forschung, grant no. 50 QI 0201 . This research was partly supported by the ESA PRODEX programme (No. 14594/00/NL/SFe) and by the Hungarian Research Fund (OTKA, No. T037508). P. Á. acknowlegdes the support of the Bolyai Fellowship. We are indebted to the referee Dr. R. J. Laureijs for useful comments, which improved the paper.

\section{References}

Abergel, A., Boulanger, F., Delouis, J. M., Duziak, G., \& Stendling, S. 1996, A\&A, 309, 245

Ábrahám, P., Leinert, Ch., \& Lemke, D. 1997, A\&A, 328, 702
Bogun, S., Lemke, D., Klaas, U., et al. 1996, A\&A, 315, 71

Chappell, D., \& Scalo, J. 2001, ApJ, 551, 712

Dame, T. M., Hartmann, D., \& Thaddeus, P. 2001, ApJ, 547, 492

Dickey, J. M., \& Lockman, F. J. 1990, ARA\&A, 28, 256

Elmegreen, B. G. 2002, ApJ, 564, 773

Gabriel, C., Acosta-Pulido, J., Heinrichsen, I., Morris, H., \& Tai, W.-M. 1997, The ISOPHOT Interactive Analysis PIA, a Calibration and Scientific Analysis Tool, in ADASS VI., ed. G. Hunt, \& H. E. Payne, ASP Conf. Ser., 125, 108

Gautier III, T. N., Boulanger, F., Pérault, M., \& Puget, J. L. 1992, AJ, 103,1313

Guiderdoni, B., Bouchet, F. R., Puget, J. L., Lagache, G., \& Hivon, E. 1997, Nature, 390, 257

Hauser, M. G., \& Dwek, E. 2001, ARA\&A, 39, 249

Helou, G., \& Beichman, C. A. 1999, The confusion limits to the sensitivity of submilimeter telescopes, in From Ground-Based to Space-Borne Sub-mm Astronomy, Proc. of the 29th Liège International Astrophysical Coll., ESA Publ., 117

Herbstmeier, U., Ábrahám, P., Lemke, D., et al. 1998, A\&A, 332, 739

Juvela, M., Mattila, K., \& Lemke, D. 2000, A\&A, 813, 360

Kessler, M. F., Steinz. J. A., Anderegg, M. F., et al. 1996, A\&A, 315, L27

Kiss, Cs., Ábrahám, P., Klaas, U., Juvela, M., \& Lemke, D. 2001, A\&A, 379, 1611

Lagache, G., Abergel, A., Boulanger, F., \& Puget, J.-L. 1998, A\&A, 333,709

Lagache, G., \& Puget, J. L. 2000, A\&A, 355, 17

Laureijs, R. J., Klaas, U., Richards, P. J., Schulz, B., \& Ábrahám, P. 2001, The ISO Handbook Vol. IV PHT - The Imaging PhotoPolarimeter, SAI-99-069/Dc, Version 2.0, Villafranca del Castillo

Lee, C. W., Myers, P. C., \& Tafalla, M. 2001, ApJ, 136, 703

Lemke, D., Klaas, U., Abolins, J., et al. 1996, A\&A, 315, L64

Low, F., Beintema, D. A., Gautier, F. N., et al. 1984, ApJ, 278, L19

Magnani, L., Blitz, L., \& Mundy, L. 1985, ApJ, 295, 402

Matsuhara, H., Kawara, K., Sato, Y., et al. 2000, A\&A, 361, 407

Meyerdierks, H., Brouillet, N., \& Mebold, U. 1990, A\&A, 230, 172

Press, W. H., Teukolsky, S. A., Vetterling, W. T., \& Flannery, B. P. 1992, Numerical Recipes in C (Cambridge University Press), 2nd edn.

Tóth, L. V., Hotzel, S., Krause, O., et al. 2000, A\&A, 364, 769

de Vries, C. P., \& van Dishoek, E. F. 1988, A\&A, 203, L23

Yonekura, Y., Dobashi, K., Mizuno, A., Ogawa, H., \& Fukui, Y. 1997, ApJS, 110, 21 